\section{Tendência temporal da prevalência de hipertensão arterial sistêmica no Brasil}

\author{
Time trend in the prevalence of systemic arterial \\ hypertension in Brazil
}

\section{Tendencia temporal de la prevalencia de hipertensión arterial sistémica en Brasil}

\section{Resumo}

Analisar as prevalências de hipertensão arterial referida por adultos brasileiros acima de 20 anos, e verificar as variáveis socioeconômicas associadas em três períodos de tempo. Os dados são da Pesquisa Nacional por Amostra de Domicílios (PNAD) realizada pelo Instituto Brasileiro de Geografia e Estatística (IBGE) nos anos de 1998 (196.439 participantes), 2003 (231.921) e 2008 (250.664). O desfecho era hipertensão arterial referida. As exposições principais eram renda em Reais e escolaridade em anos de estudos. A análise dos dados foi feita usando-se regressão de Poisson com variância robusta com controle para amostras complexas. Maiores prevalências de hipertensão arterial foram encontradas em pessoas com menor escolaridade, independentemente dos anos estudados e do sexo. Baixa renda esteve associada a maiores prevalências de hipertensão arterial independentemente dos anos estudados na amostra total e nas mulheres. Dentre os homens, esse efeito não foi verificado nos anos de 1998 e 2003. Já em 2008, homens de alta renda apresentaram maiores prevalências de hipertensão arterial, sugerindo modificação de efeito. Dessa forma, o presente trabalho apontou o aumento das prevalências de hipertensão arterial nos períodos estudados, destacando sua associação negativa com aspectos socioeconômicos.

Hipertensão; Desigualdades em Saúde; Inquéritos Epidemiológicos
Larissa Aline Carneiro Lobo 1

Raquel Canuto 1,2

Juvenal Soares Dias-da-Costa 1

Marcos Pascoal Pattussi 1

doi: 10.1590/0102-311X00035316

\section{Correspondência}

L. A. C. Lobo

Universidade do Vale do Rio dos Sinos.

Av. Unisinos 950, São Leopoldo, RS 93022-000, Brasil.

lalilobo@yahoo.com.br

1 Universidade do Vale do Rio dos Sinos, São Leopoldo, Brasil. 2 Departamento de Nutrição, Universidade Federal do Rio Grande do Sul, Porto Alegre, Brasil. 


\section{Introdução}

As doenças crônicas não transmissíveis (DCNTs) podem ser consideradas como problema de saúde pública no Brasil e no mundo 1,2. De acordo com dados da Organização Mundial da Saúde (OMS), das DCNTs, as doenças do aparelho circulatório são responsáveis por cerca de 17 milhões de mortes/ano em todo o mundo. Dessas, 55,3\% corresponderam a complicações decorrentes da hipertensão arterial. Em 2008, cerca de 40\% dos adultos com 25 anos ou mais foram diagnosticados com hipertensão arterial, sendo que a doença correspondeu a $12,8 \%$ do total de mortes anuais, o que representou $3,7 \%$ do total de carga de doença (disability adjusted life of years - DALY) 2,3.

Embora, a hipertensão arterial seja um reconhecido fator de risco para a morbidade e mortalidade para doenças do aparelho circulatório, estudos com representatividade nacional são escassos em países em desenvolvimento 4 . Uma revisão sistemática da literatura, com 14 trabalhos, totalizando 17.085 indivíduos em diferentes cidades brasileiras, encontrou uma prevalência de hipertensão arterial igual a 28,7\% entre os anos 2000 e 2007 5. Em um inquérito telefônico nacional 6, a prevalência na população brasileira para o ano de 2011 foi de 24,8\%. Por sua vez, a Pesquisa Nacional de Saúde encontrou prevalência de hipertensão arterial de 21,4\%, elevando-se de acordo com o aumento da idade, maior nos indivíduos de baixa escolaridade, do sexo feminino e nas pessoas de raça/cor da pele preta 45 .

Entretanto, a distribuição da hipertensão arterial não ocorre igualmente entre as regiões e cidades brasileiras. Em todo o mundo a literatura demonstra que o risco de apresentar doenças do aparelho circulatório é maior nos grupos menos favorecidos 8,9,10. No Brasil, existem estudos que mostram as tendências das prevalências de hipertensão arterial 5,11, porém tais estudos não abordam as influências das variáveis socioeconômicas com o decorrer do tempo. Portanto, o presente trabalho tem como objetivo analisar as prevalências de hipertensão arterial referida por adultos brasileiros, e verificar as variáveis socioeconômicas associadas durante três períodos de tempo.

\section{Métodos}

Este estudo utilizou dados secundários de domínio público da Pesquisa Nacional por Amostra de Domicílios (PNAD) realizada nos anos de 1998, 2003 e 2008. A PNAD é realizada no Brasil desde 1967 pelo Instituto Brasileiro de Geografia e Estatística (IBGE). São inquéritos epidemiológicos de base populacional com abrangência nacional, que tem como objetivo coletar informações sobre as características demográficas, habitação, educação, trabalho e rendimentos da população brasileira 12. Os inquéritos ocorrem anualmente e os dados são coletados por meio de entrevistas estruturadas, conduzidas no domicílio dos participantes.

A PNAD tem sido realizada por intermédio de uma amostra probabilística de domicílios obtida em três estágios de seleção: unidades primárias - municípios; unidades secundárias - setores censitários; e unidades terciárias - unidades domiciliares (domicílios particulares e unidades de habitação em domicílios coletivos) 12 .

No primeiro estágio, as unidades (municípios) foram classificadas em duas categorias: autorrepresentativas (probabilidade 1 de pertencer à amostra) e não autorrepresentativas. Os municípios pertencentes à segunda categoria passaram por um processo de estratificação e, em cada estrato, foram selecionados com reposição e com probabilidade proporcional à população residente obtida no Censo Demográfico do ano em questão 12 .

No segundo, as unidades (setores censitários) foram selecionadas, em cada município da amostra, também com probabilidade proporcional e com reposição, sendo utilizado o número de unidades domiciliares existentes por ocasião do Censo Demográfico como medida de tamanho 12

No último estágio foram selecionados, com equiprobabilidade, em cada setor censitário da amostra, os domicílios particulares e as unidades de habitação em domicílios coletivos para investigação das características dos moradores e da habitação. Foram excluídas as pessoas residentes em embaixadas, consulados e delegações e, também, as pessoas institucionalizadas, residentes em domicílios coletivos de estabelecimentos institucionais 12 .

Suplementos especiais que investigam outras características populacionais têm sido adicionados à PNAD com periodicidade variável de acordo com a necessidade de informação do país ${ }^{12}$. Nos anos de 
1998, 2003 e 2008 o suplemento especial da PNAD contemplou o tema saúde, com informações relativas às condições de saúde autorreferidas, uso de serviços de saúde e aspectos relacionados. O número de pessoas que participaram da PNAD nesses anos foi 344.975, 384.834 e 391.868, respectivamente 12. No presente artigo foram analisados apenas os dados dos participantes com idade superior a 20 anos, correspondendo a 196.439 em 1998, 231.921 em 2003 e 250.664 em 2008.

O desfecho utilizado foi hipertensão arterial referida. No ano de 1998, essa variável foi coletada usando-se a pergunta “tem hipertensão (pressão alta)?". Já nos anos de 2003 e 2008, a pergunta sofreu modificações, sendo questionado se "algum médico ou profissional da saúde disse que você tem hipertensão (pressão alta)?” 12. Mudanças na formulação das questões podem provocar problemas na comparabilidade entre os trabalhos. Entretanto, informações autorreferidas de hipertensão arterial em estudos de base populacional têm sido consideradas válidas para o entendimento da condição 7 .

Foram incluídas na análise as variáveis demográficas: sexo (masculino/feminino); idade (21-39 anos, 40-59 e 60 anos ou mais); cor da pele (branca, preta, amarela, parda e indígena) e regiões do Brasil (Norte, Nordeste, Sul, Sudeste, Centro-oeste).

As exposições principais foram renda e escolaridade. A variável renda foi calculada com base na variável renda mensal domiciliar, fornecida pelo próprio IBGE, que corresponde ao valor do rendimento mensal domiciliar para todas as unidades domiciliares (excluindo o rendimento dos pensionistas, empregados domésticos, parentes dos empregados domésticos e pessoas com menos de 10 anos de idade). Os valores foram transformados em salários mínimos (SM) vigentes no ano das pesquisas e categorizados em faixas de salários mínimos ${ }^{13,14}$. O salário mínimo correspondia a $R \$ 130,00$ em 1998, R\$ 240,00 em 2003 e R \$ 415,00 em 2008, e foi categorizado em três faixas: alta (> 10,0 SM), média $(3,0-10,0$ SM) e baixa $(<3,0$ SM).

A escolaridade foi analisada por meio da variável "anos de estudos", disponibilizada pelo próprio IBGE, que foi obtida em função da série e do nível ou grau que a pessoa estava frequentando ou havia frequentado, considerando-se a última série concluída com aprovação. A variável foi agrupada em cinco faixas de escolaridade, de acordo com o sistema de educação brasileiro: menos de 1 ano (analfabeto); 1-4 anos (Ensino Fundamental incompleto); 5-8 (Ensino Fundamental completo); 9-11 (Ensino Médio incompleto); 12 ou mais (Ensino Médio completo).

A análise dos dados foi conduzida no programa Stata 11.0 (Stata Corp. College Station, Estados Unidos). Inicialmente, foram descritas as características da amostra. A análise foi estratificada por sexo, mostraram-se as prevalências de hipertensão arterial com os respectivos intervalos de $95 \%$ de confiança (IC95\%) para as categorias das variáveis independentes nos três anos de estudo. Para fornecer uma estimativa das razões de prevalências (RP) brutas e ajustadas, além de seus respectivos IC95\%, utilizou-se a regressão de Poisson com variância robusta, com controle para amostras complexas. A entrada das variáveis no modelo multivariável foi simultânea, de forma que o efeito das exposições foi ajustado para as variáveis demográficas (idade, cor da pele e regiões do Brasil), coletadas nos três anos analisados. Por último, testes de interação revelaram modificação efeito entre hipertensão arterial, sexo e renda/escolaridade, sendo realizada a estratificação por sexo.

\section{Resultados}

Um total de 196.439 pessoas com idade superior a 20 anos participaram da PNAD em 1998, 231.921 em 2003 e 250.664 em 2008. As prevalências de hipertensão arterial referida foram de 18,0\% (IC95\%: 17,9-18,2) em 1998, 19,2\% (IC95\%: 19,0-19,4) em 2003 e 20,9\% (IC95\%: 20,7-21,0) em 2008, ou seja, aumentaram nos períodos estudados, sendo sempre mais elevadas dentre as mulheres (Figura 1).

A amostra, em todos os anos estudados, foi constituída em sua maioria de mulheres, de pessoas com idades entre 21 a 39 anos e de cor da pele branca. As regiões Sudeste e Nordeste foram as mais representadas nos três estudos. Quanto à escolaridade, observou-se o predomínio dos níveis Fundamental incompleto, Fundamental completo e Médio incompleto. Em relação à renda, em 1998 predominou a de três até dez SM. Nos dois últimos anos, a maioria da população referiu receber renda inferior a três SM (Tabela 1).

As prevalências de hipertensão arterial de acordo com sexo, idade, cor da pele, regiões do Brasil, escolaridade e renda estão apresentadas na Tabela 2. Nos três anos estudados observaram-se 
Tendência temporal da prevalência de hipertensão arterial na população total, nos homens e nas mulheres com mais de 20 anos de idade. Pesquisa Nacional por Amostra de Domicílios (PNAD) 1998, 2003 e 2008, Brasil.

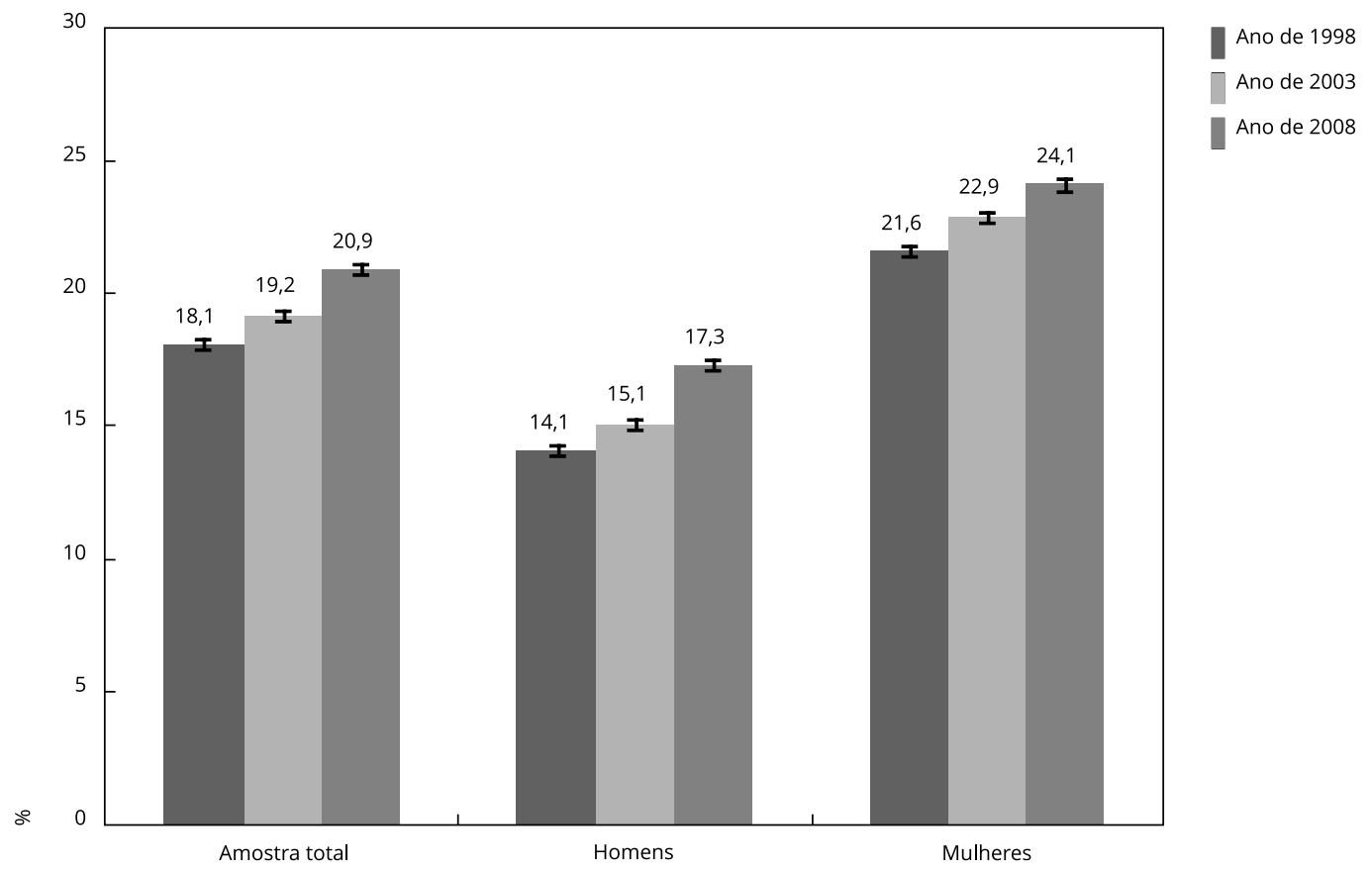

tendência linear direta entre idade e hipertensão arterial, ou seja, as prevalências do desfecho aumentaram de acordo com as mudanças de faixas etárias. Foram encontradas maiores prevalências de hipertensão arterial nos indivíduos de cor da pele preta, nos índios e nas pessoas de coloração amarela, inclusive na estratificação, nos três períodos de tempo. Na distribuição por regiões do Brasil, observaram-se maiores prevalências de hipertensão arterial nas regiões Sudeste e Sul, e entre os homens e as mulheres, para os três anos de estudo (Tabela 2).

Verificaram-se também maiores prevalências de hipertensão arterial nas pessoas com menor escolaridade, independentemente dos anos estudados e do sexo. Observou-se que as prevalências aumentaram nos anos estudados para as pessoas de menor escolaridade. Por exemplo, a prevalência de hipertensão arterial referida entre os analfabetos era cerca de 30\% no ano de 1998, chegando a 36,4\% em 2008. Esse padrão repetiu entre homens e mulheres. Com relação à renda, maiores prevalências de hipertensão arterial foram observadas nos indivíduos do grupo de menor renda. Na análise estratificada por sexo, verificou-se que nas mulheres de menor renda as prevalências de hipertensão arterial foram maiores. Dentre os homens nos anos de 2003 e 2008, as maiores prevalências de hipertensão arterial foram observadas no grupo de maior renda (Tabela 2).

$\mathrm{Na}$ análise bruta, os menores níveis de escolaridade estavam associados com maiores prevalências de hipertensão arterial referida na amostra total e em ambos os sexos, independentemente dos anos estudados. As maiores magnitudes foram encontradas dentre as mulheres. Razões de prevalência cerca de quatro vezes maiores foram encontradas nas mulheres analfabetas, quando comparadas àquelas com ensino médio completo ou mais. Na análise ajustada, após o controle para variáveis demográficas (idade, cor da pele e região do Brasil), a magnitude reduziu pela metade, mas, ainda assim, foi mantida na mesma direção. A mesma associação foi encontrada nos homens, embora com menor magnitude (Tabela 3). 


\section{Tabela 1}

Descrição das características demográficas e socioeconômicas na população total, nos homens e nas mulheres com mais de 20 anos de idade. Pesquisa Nacional por Amostra de Domicílios (PNAD), 1998, 2003 e 2008, Brasil.

\begin{tabular}{|c|c|c|c|c|c|c|c|c|c|}
\hline & \multicolumn{3}{|c|}{ Amostra total (\%) } & \multicolumn{3}{|c|}{ Homens (\%) } & \multicolumn{3}{|c|}{ Mulheres (\%) } \\
\hline & 1998 & 2003 & 2008 & 1998 & 2003 & 2008 & 1998 & 2003 & 2008 \\
\hline Total (n) & 196.439 & 231.921 & 250.664 & 93.204 & 109.838 & 118.552 & 103.512 & 122.083 & 132.112 \\
\hline \multicolumn{10}{|l|}{ Hipertensão } \\
\hline Não & 81,9 & 80,8 & 79,1 & 85,9 & 84,9 & 82,7 & 78,4 & 77,1 & 75,9 \\
\hline Sim & 18,1 & 19,2 & 20,9 & 14,1 & 15,1 & 17,3 & 21,6 & 22,9 & 24,1 \\
\hline \multicolumn{10}{|l|}{ Idade (anos) } \\
\hline $21-39$ & 52,4 & 51,3 & 52,4 & 53,2 & 52,5 & 53,2 & 51,6 & 50,1 & 51,6 \\
\hline $40-59$ & 32,9 & 33,6 & 32,9 & 32,9 & 33,4 & 33,1 & 32,7 & 33,4 & 32,7 \\
\hline 60 ou mais & 14,7 & 15,1 & 14,7 & 14,7 & 14,0 & 13,7 & 15,6 & 16,1 & 15,6 \\
\hline \multicolumn{10}{|l|}{ Cor da pele } \\
\hline Índio & 0,2 & 0,2 & 0,4 & 0,23 & 0,23 & 0,34 & 0,20 & 0,23 & 0,36 \\
\hline Branca & 53,7 & 50,0 & 46,5 & 52,62 & 48.9 & 45,1 & 54,8 & 50,9 & 47,6 \\
\hline Preta & 6,4 & 6,9 & 8,2 & 6,57 & 7,2 & 8,43 & 6,26 & 6,67 & 7,95 \\
\hline Amarela & 0,5 & 0,4 & 0,6 & 0,49 & 0,39 & 0,51 & 0,48 & 0,46 & 0,59 \\
\hline Parda & 39,2 & 42,5 & 44,4 & 40,08 & 43,3 & 45,5 & 38,4 & 41,7 & 43,4 \\
\hline \multicolumn{10}{|l|}{ Região do Brasil } \\
\hline Norte & 6,54 & 10,1 & 11,8 & 6,80 & 10,2 & 12,2 & 6,69 & 9,94 & 11,4 \\
\hline Nordeste & 30,0 & 31,1 & 30,8 & 29,5 & 30,9 & 30,6 & 30,4 & 31,2 & 31,1 \\
\hline Sudeste & 35,1 & 31,5 & 30,9 & 35,1 & 31,3 & 30,6 & 35,1 & 31,8 & 31,1 \\
\hline Sul & 17,6 & 16,5 & 15,4 & 17,8 & 16.5 & 15.5 & 17,4 & 16,4 & 15,4 \\
\hline Centro-oeste & 10,6 & 10,9 & 11,0 & 10,8 & 11,1 & 11,1 & 10,4 & 10,7 & 10,9 \\
\hline \multicolumn{10}{|l|}{ Escolaridade } \\
\hline$\geq$ Ensino Médio completo & 9,6 & 11,2 & 14,3 & 9,40 & 10,3 & 13,0 & 9,69 & 11,9 & 15,4 \\
\hline Ensino Médio incompleto & 18,9 & 24,4 & 29,2 & 18,1 & 23,6 & 28,8 & 19,7 & 25.0 & 29,6 \\
\hline Ensino Fundamental completo & 24,4 & 23,7 & 22,0 & 25,1 & 24,7 & 23,0 & 23,8 & 22,8 & 21,1 \\
\hline Ensino Fundamental incompleto & 30,2 & 25,7 & 21,6 & 30,6 & 26,2 & 22,2 & 29,8 & 25,2 & 21,1 \\
\hline Analfabeto & 16,5 & 14,4 & 12,5 & 16,4 & 14,5 & 12,7 & 16,5 & 14,4 & 12,3 \\
\hline \multicolumn{10}{|l|}{ Renda mensal (SM) * } \\
\hline$<3,0$ & 34,4 & 45,3 & 46,4 & 33,5 & 44,7 & 39,9 & 35,2 & 45,9 & 47,1 \\
\hline$>3,0-10,0$ & 41,0 & 38,7 & 39,3 & 41,5 & 39,2 & 45,5 & 40,6 & 38,3 & 38,7 \\
\hline$>10,1$ & 24,6 & 15,9 & 14,3 & 25,0 & 16,1 & 14,5 & 24,2 & 15,8 & 14,1 \\
\hline
\end{tabular}

SM: salários mínimos.

* Renda mensal em SM para o ano de 1998 (R\$130,00), 2003 (R\$240,00) e $2008(R \$ 415,00)$.

Quanto à renda, tanto na análise bruta quanto na ajustada, as prevalências de hipertensão arterial eram maiores nos grupos menos favorecidos independentemente dos anos estudados na amostra total e nas mulheres. Essa diferença não foi verificada entre os homens nos anos de 1998 e 2003 . Nesses dois períodos, não foram encontradas diferenças nas prevalências de hipertensão arterial entre os indivíduos de maior e de menor nível de renda. Já em 2008, a análise mostrou que a prevalência de hipertensão arterial foi menor na categoria de menor renda, ainda que o limite superior do intervalo de confiança quase tenha atingido o valor unitário, sugerindo modificação de efeito. 


\section{Tabela 2}

Prevalências da hipertensão arterial na população total, nos homens e nas mulheres de acordo com as variáveis demográficas e socioeconômicas. Pesquisa Nacional por Amostra de Domicílios (PNAD), 1998 ( $n=196.439), 2003(n=231.921)$ e 2008 ( $n=250.664)$, Brasil.

\begin{tabular}{|c|c|c|c|}
\hline & $\begin{array}{c}1998 \\
\% \text { (IC95\%) }\end{array}$ & $\begin{array}{c}2003 \\
\% \text { (IC95\%) }\end{array}$ & $\begin{array}{c}2008 \\
\% \text { (IC95\%) }\end{array}$ \\
\hline \multicolumn{4}{|l|}{ Amostra total } \\
\hline \multicolumn{4}{|l|}{ Idade (anos) } \\
\hline 21-39 & $6,5(6,3-6,6)$ & $6,3(6,1-6,4)$ & $6,3(6,1-6,4)$ \\
\hline $40-59$ & $24,8(24,5-25,1)$ & $25,7(25,4-26,0)$ & $26,9(26,7-27,3)$ \\
\hline 60 ou mais & $44,1(43,5-44,7)$ & $48,6(48,0-49,1)$ & $53,2(52,7-53,7)$ \\
\hline \multicolumn{4}{|l|}{ Cor da pele } \\
\hline Índio & $16,7(13,2-20,3)$ & $23,5(19,9-27,2)$ & $24,7(21,8-27,6)$ \\
\hline Branca & $17,5(17,3-17,7)$ & $19,3(19,1-19,5)$ & $21,2(20,9-21,4)$ \\
\hline Preta & $24,4(23,6-25,1)$ & $24,1(23,4-24,7)$ & $25,2(24,6-25,8)$ \\
\hline Amarela & $17,6(15,2-20,1)$ & $19,5(17,6-22,0)$ & $23,2(20,9-25,4)$ \\
\hline Parda & $17,7(17,5-18,0)$ & $18,3(18,0-18,5)$ & $19,7(19,4-19,9)$ \\
\hline \multicolumn{4}{|l|}{ Região do Brasil } \\
\hline Norte & $15,5(14,9-16,1)$ & $14,8(14,3-15,2)$ & $15,7(15,2-16,1)$ \\
\hline Nordeste & $17,9(17,6-18,2)$ & $18,3(18,1-18,6)$ & $19,9(19,7-20,2)$ \\
\hline Sudeste & $18,8(18,5-19,1)$ & $21,2(20,9-21,5)$ & $23,5(23,2-23,8)$ \\
\hline Sul & $18,1(17,7-18,5)$ & $20,0(19,6-20,4)$ & $22,2(21,8-22,6)$ \\
\hline Centro-oeste & $17,3(16,8-17,8)$ & $18,7(18,2-19,2)$ & $19,9(19,4-20,3)$ \\
\hline \multicolumn{4}{|l|}{ Escolaridade } \\
\hline z Ensino Médio completo & $11,0(10,2-11,0)$ & $12,0(11,6-12,4)$ & $13,3(13,0-13,6)$ \\
\hline Ensino Médio incompleto & $10,0(9,70-10,3)$ & $10,5(10,2-10,7)$ & $12,7(12,4-13,0)$ \\
\hline Ensino Fundamental completo & $14,0(13,6-14,2)$ & $16,1(15,7-16,4)$ & $18,4(18,0-18,7)$ \\
\hline Ensino Fundamental incompleto & $22,4(22,0-22,7)$ & $26,0(25,6-26,4)$ & $30,9(30,4-31,2)$ \\
\hline Analfabeto & $30,0(29,4-30,5)$ & $32,9(32,4-33,5)$ & $36,4(36,0-37,0)$ \\
\hline \multicolumn{4}{|l|}{ Renda mensal (SM) * } \\
\hline$<3,0$ & $20,1(19,8-20,4)$ & $20,4(17,0-17,8)$ & $21,6(21,4-21,8)$ \\
\hline$>3,0-10,0$ & $17,6(17,3-17,9)$ & $18,6(17,8-18,5)$ & $20,4(20,1-20,6)$ \\
\hline$>10,01$ & $15,8(15,5-16,1)$ & $17,4(17,0-17,8)$ & $20,0(19,6-20,4)$ \\
\hline
\end{tabular}

(continua)

\section{Discussão}

Uma das limitações mais importantes deste trabalho refere-se ao fato de utilizar dados referidos ao invés de observados. Entretanto, estudos realizados no Brasil atestam uma boa validade do autorrelato de hipertensão arterial, com sensibilidade que varia de 71,1\% a 84,3\% e especificidade de $80,5 \%$ a $87,5 \%$. Além disso, trata-se de uma estratégia rápida, barata e acessível, podendo ser considerado um bom método de monitorização da hipertensão arterial, complementarmente ao método de aferição em $\mathrm{mm} / \mathrm{Hg} 15,16,17$.

Os resultados deste estudo demonstram que a prevalência de hipertensão arterial vem aumentando ao longo dos anos. Segundo dados da OMS 2, o aumento da prevalência de hipertensão arterial pode ser atribuído ao crescimento da população mundial, ao envelhecimento populacional, além da exposição a comportamentos de risco, como maus hábitos alimentares, consumo de álcool e tabaco, e exposição crônica ao estresse. Além disso, recente atualização apontou que as prevalências de níveis tensionais elevados são mais prevalentes entre as mulheres, nos indivíduos de idade mais avançada, de cor da pele preta, com menores níveis de escolaridade e de renda 18 . 


\begin{tabular}{|c|c|c|c|}
\hline & $\begin{array}{c}1998 \\
\% \text { (IC95\%) }\end{array}$ & $\begin{array}{c}2003 \\
\% \text { (IC95\%) }\end{array}$ & $\begin{array}{c}2008 \\
\% \text { (IC95\%) }\end{array}$ \\
\hline \multicolumn{4}{|l|}{ Homens } \\
\hline \multicolumn{4}{|l|}{ Idade (anos) } \\
\hline 21-39 & $4,9(4,7-5,1)$ & $4,8(4,6-4,9)$ & $5,3(5,2-5,5)$ \\
\hline $40-59$ & $19,5(19,0-19,9)$ & $20,8(20,4-21,2)$ & $22,7(22,3-23,1)$ \\
\hline 60 ou mais & $36,8(35,9-37,6)$ & $40,4(39,6-41,1)$ & $46,1(45,3-46,8)$ \\
\hline \multicolumn{4}{|l|}{ Cor da pele } \\
\hline Índio & $15,1(10,3-19,9)$ & $20,6(15,5-25,6)$ & $20,7(16,7-24,6)$ \\
\hline Branca & $14,3(14,0-14,6)$ & $15,8(15,5-16,1)$ & $18,4(18,0-18,7)$ \\
\hline Preta & $18,3(17,3-19,2)$ & $18,2(17,4-19,1)$ & $19,7(18,8-20,4)$ \\
\hline Amarela & $15,9(12,6-19,3)$ & $15,7(12,3-19,2)$ & $21,2(17,9-24,5)$ \\
\hline Parda & $13,1(12,8-13,4)$ & $13,9(13,5-14,2)$ & $15,7(15,4-16,0)$ \\
\hline \multicolumn{4}{|l|}{ Região do Brasil } \\
\hline Norte & $11.7(10,9-12,5)$ & $11,8(11,2-12,3)$ & $12,9(12,4-13,5)$ \\
\hline Nordeste & $13,2(12,8-13,6)$ & $13,7(13,4-14,1)$ & $15,8(15,4-16,1)$ \\
\hline Sudeste & $15,3(14,9-15,7)$ & $16,9(16,5-17,3)$ & $19,9(19,5-20,3)$ \\
\hline Sul & $14,2(13,7-14,8)$ & $16,1(15,6-16,7)$ & $18,8(18,2-19,4)$ \\
\hline Centro-oeste & $14,1(13,4-14,8)$ & $15,7(15,0-16,3)$ & $17,0(16,4-17,7)$ \\
\hline \multicolumn{4}{|l|}{ Escolaridade } \\
\hline z Ensino Médio completo & $12,8(12,1-13,5)$ & $13,8(13,2-14,5)$ & $14,9(14,4-15,5)$ \\
\hline Ensino Médio incompleto & $9,26(8,8-9,70)$ & $9,63(9,2-9,9)$ & $11,7(11,3-12,0)$ \\
\hline Ensino Fundamental completo & $10,8(10,4-11,2)$ & $12,0(11,6-12,4)$ & $14,6(14,2-15,1)$ \\
\hline Ensino Fundamental incompleto & $16,6(16,2-17,1)$ & $19,3(18,9-19,8)$ & $23,4(22,9-23,9)$ \\
\hline Analfabeto & $20,7(20,0-21,3)$ & $23,1(22,4-23,7)$ & $26,7(26,0-27,4)$ \\
\hline \multicolumn{4}{|l|}{ Renda mensal (SM) * } \\
\hline$<3,0$ & $14,5(14,1-14,9)$ & $14,9(14,6-15,2)$ & $16,6(16,3-16,9)$ \\
\hline$>3,0-10,0$ & $13,5(13,2-13,8)$ & $14,9(14,6-15,2)$ & $17,3(16,9-17,6)$ \\
\hline$>10,01$ & $14,5(14,0-14,9)$ & $16,5(15,9-17,0)$ & $19,5(18,9-20,1)$ \\
\hline
\end{tabular}

(continua)

Nas últimas décadas, o Brasil vem passando por um processo de transição demográfica, epidemiológica e social. Uma das consequências dessa mudança é o aumento da longevidade populacional e aumento das taxas de morbidade e mortalidade da população. O envelhecimento da população acarreta um significativo crescimento da carga das doenças cardiovasculares 9,19. Neste trabalho, nos três anos estudados, observou-se uma tendência linear direta entre aumento das faixas de idade da população e maiores prevalências de hipertensão arterial.

Essas maiores prevalências foram relatadas nas regiões Sudeste e Sul do Brasil. Esses achados podem estar relacionados a um maior acesso a serviços de saúde nessas regiões e, consequentemente, maior conhecimento de sua condição de saúde por estes indivíduos 7,20,21.

O perfil de morbidade, tanto para doenças crônicas quanto para as agudas, é distinto entre homens e mulheres 22. Neste estudo, maiores prevalências de hipertensão arterial foram encontradas em mulheres em todos os anos analisados. Fatores de risco como obesidade, menor acesso às condições materiais e sociais, estresse e pressões relacionadas ao papel exercido pela mulher aumentariam o risco de doenças neste grupo. Já os homens, estariam mais susceptíveis a fatores relacionados ao uso de álcool e tabaco, e a situações de risco relacionadas ao trabalho. De maneira geral, mulheres utilizam mais os serviços de saúde, preocupam-se mais com o seu próprio estado de saúde e relatam mais problemas de saúde do que os homens 22,23,24,25,26,27.

Neste trabalho, maiores prevalências de hipertensão arterial foram encontradas em pessoas com menor escolaridade, independentemente dos anos estudados e do sexo. Menor renda esteve associada 


\begin{tabular}{|c|c|c|c|}
\hline & $\begin{array}{c}1998 \\
\%(\mathrm{IC} 95 \%)\end{array}$ & $\begin{array}{c}2003 \\
\%(I C 95 \%)\end{array}$ & $\begin{array}{c}2008 \\
\%(\mathrm{IC} 95 \%)\end{array}$ \\
\hline \multicolumn{4}{|l|}{ Mulheres } \\
\hline \multicolumn{4}{|l|}{ Idade (anos) } \\
\hline 21-39 & $7,9(7,7-8,1)$ & $7,6(7,4-7,8)$ & $7,1(6,9-7,3)$ \\
\hline $40-59$ & $29,7(29,2-30,1)$ & $30,3(29,7-30,6)$ & $30,8(30,4-31,2)$ \\
\hline 60 ou mais & $49,9(49,1-50,7)$ & $55,0(54,3-55,7)$ & $58,7(58,1-59,3)$ \\
\hline \multicolumn{4}{|l|}{ Cor da pele } \\
\hline Índio & $18,4(13,1-23,6)$ & $26,1(20,9-31,3)$ & $28,2(24,1-32,2)$ \\
\hline Branca & $20,3(19,9-20,6)$ & $22,3(21,9-22,6)$ & $23,6(23,3-23,9)$ \\
\hline Preta & $30,2(20,1-31,3)$ & $29,7(28,7-30,7)$ & $30,5(29,6-31,4)$ \\
\hline Amarela & $19,2(15,7-22,7)$ & $22,5(19,0-25,9)$ & $24,7(21,7-27,7)$ \\
\hline Parda & $22,1(21,6-22,5)$ & $22,4(22,0-22,8)$ & $23,4(23,0-23,7)$ \\
\hline \multicolumn{4}{|l|}{ Região do Brasil } \\
\hline Norte & $18,9(18,0-19,9)$ & $17,6(16,9-18,3)$ & $18,3(17,7-18,9)$ \\
\hline Nordeste & $22,0(21,6-22,5)$ & $22,5(22,0-22,9)$ & $23,7(23,2-24,1)$ \\
\hline Sudeste & $22,0(21,6-22,4)$ & $25,0(24,6-25,4)$ & $26,7(26,2-27,1)$ \\
\hline Sul & $21,7(21,1-22,3)$ & $23,6(22,3-24,1)$ & $25,3(24,7-25,9)$ \\
\hline Centro-oeste & $20,3(19,5-21,0)$ & $21,5(20,8-22,2)$ & $22,4(21,8-23,1)$ \\
\hline \multicolumn{4}{|l|}{ Escolaridade } \\
\hline 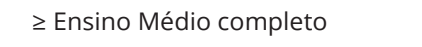 & $8,66(8,1-9,2)$ & $10,5(10,0-11,0)$ & $11,9(11,5-12,4)$ \\
\hline Ensino Médio incompleto & $10,6(10,2-11,1)$ & $11,2(10,8-11,5)$ & $13,5(13,1-13,8)$ \\
\hline Ensino Fundamental completo & $16,9(16,4-17,3)$ & $19,9(19,5-20,4)$ & $22,0(21,5-22,5)$ \\
\hline Ensino Fundamental incompleto & $27,7(27,2-28,2)$ & $32,3(31,8-32,8)$ & $37,7(37,1-38,3)$ \\
\hline Analfabeto & $38,3(37,6-39,0)$ & $41,9(41,2-42,7)$ & $45,4(44,6-46,2)$ \\
\hline \multicolumn{4}{|l|}{ Renda mensal (SM) * } \\
\hline$<3,0$ & $24,9(24,5-25,4)$ & $25,3(24,9-25,7)$ & $25,9(25,6-26,3)$ \\
\hline$>3,0-10,0$ & $21,4(21,0-21,8)$ & $22,0(21,6-22,4)$ & $23,3(22,9-23,7)$ \\
\hline$>10,01$ & $17,0(16,5-17,5)$ & $18,3(17,7-18,8)$ & $20,5(19,9-21,1)$ \\
\hline
\end{tabular}

IC95\%: intervalo de 95\% de confiança; SM: salários mínimos.

* Renda mensal em SM para o ano de 1998 (R\$130,00), 2003 (R\$240,00) e $2008(R \$ 415,00)$.

a maiores prevalências de hipertensão arterial na amostra total e dentre as mulheres, em todos os anos estudados. A associação entre baixa escolaridade e baixa renda com hipertensão arterial é vastamente citada nas literaturas internacional 8,10,28,29,30,31,32,33,34,35,36 e nacional 37,38,39,40,41,42.

Para os homens, no ano de 2008, maiores níveis de renda estiveram associados a maiores prevalências de hipertensão arterial, sugerindo modificação de efeito ao se comparar com resultados verificados entre as mulheres. De forma similar aos resultados encontrados por Marins et al. 43 , em que foi verificada uma associação inversa entre renda per capita (< USD 200/mês) e o risco de apresentar doença cardiovascular (odds ratio - OR = 0,77; IC95\%: 0,61-0,97). Entretanto, os autores ressaltam que a associação entre renda e risco cardiovascular não é clara. Segundo Kaplan \& Keil 44, a mensuração da variável renda é complexa e o nível de renda pode variar ao longo do tempo. Da mesma forma, para Luepker et al. 31 o nível educacional, entre as variáveis socioeconômicas, é o principal fator de risco para a hipertensão arterial já que a renda pode ser modificada ao longo da vida, não sendo um bom indicador para fatores de risco cardiovascular. Já de acordo com Luoto et al. 32 e Gaudemaris et al. 30, a renda pode ser considerada um bom indicador socioeconômico apenas entre as mulheres.

Para explicar o mecanismo de ação das desigualdades socioeconômicas sobre a situação de saúde, Towsend et al. 33 sugerem uma série de teorias. Tentam explicar as desigualdades em saúde como "artefato", como "seleção natural e social”, como uma forma "materialista ou estruturalista" ou ainda como uma "perspectiva cultural". Os achados do presente estudo não podem ser explicados pela 
Razões de prevalências (RP) bruta e ajustada da hipertensão em relação às variáveis socioeconômicas na população total, nos homens e nas mulheres. Pesquisa Nacional por Amostra de Domicílios (PNAD), $1998(n=196.439), 2003(n=231.921)$ e $2008(n=250.664)$.

\begin{tabular}{|c|c|c|c|c|c|c|}
\hline & \multicolumn{2}{|c|}{1998} & \multicolumn{2}{|c|}{2003} & \multicolumn{2}{|c|}{2008} \\
\hline & $\begin{array}{l}\text { RP bruta } \\
\text { (IC95\%) }\end{array}$ & $\begin{array}{l}\text { RP ajustada * } \\
\text { (IC95\%) }\end{array}$ & $\begin{array}{l}\text { RP bruta } \\
\text { (IC95\%) }\end{array}$ & $\begin{array}{l}\text { RP ajustada * } \\
\text { (IC95\%) }\end{array}$ & $\begin{array}{l}\text { RP bruta } \\
\text { (IC95\%) }\end{array}$ & $\begin{array}{l}\text { RP ajustada * } \\
\text { (IC95\%) }\end{array}$ \\
\hline \multicolumn{7}{|l|}{ Amostra total } \\
\hline \multicolumn{7}{|l|}{ Escolaridade } \\
\hline $\begin{array}{l}\geq \text { Ensino Médio } \\
\text { completo }\end{array}$ & 1,00 & 1,00 & 1,00 & 1,00 & 1,00 & 1,00 \\
\hline $\begin{array}{l}\text { Ensino Médio } \\
\text { incompleto }\end{array}$ & $0,94(0,89-0,99)$ & $1,10(1,05-1,16)$ & $0,87(0,84-0,91)$ & $1,05(1,01-1,09)$ & $0,95(0,92-0,98)$ & $1,11(1,08-1,15)$ \\
\hline $\begin{array}{l}\text { Ensino Fundamental } \\
\text { completo }\end{array}$ & $1,31(1,25-1,38)$ & $1,39(1,33-1,45)$ & $1,34(1,29-1,39)$ & $1,37(1,33-1,42)$ & $1,38(1,34-1,43)$ & $1,32(1,28-1,36)$ \\
\hline $\begin{array}{l}\text { Ensino Fundamental } \\
\text { incompleto }\end{array}$ & $2,11(2,02-2,20)$ & $1,58(1,52-1,65)$ & $2,17(2,09-2,25)$ & $1,54(1,49-1,59)$ & $2,32(2,25-2,39)$ & $1,47(1,43-1,51)$ \\
\hline Analfabeto & $2,83(2,70-2,96)$ & $1,64(1,56-1,71)$ & $2,75(2,65-2,85)$ & $1,56(1,51-1,62)$ & $2,74(2,66-2,83)$ & $1,47(1,42-1,51)$ \\
\hline \multicolumn{7}{|l|}{ Renda mensal (SM) ** } \\
\hline$>10,01$ & 1,00 & 1,00 & 1,00 & 1,00 & 1,00 & 1,00 \\
\hline$>3,0-10,0$ & $1,11(1,08-1,14)$ & $1,17(1,14-1,19)$ & $1,07(1,04-1,09)$ & $1,13(1,10-1,16)$ & $1,02(0,99-1,04)$ & $1,08(1,06-1,11)$ \\
\hline$<3,0$ & $1,27(1,24-1,31)$ & $1,25(1,22-1,28)$ & $1,17(1,14-1,20)$ & $1,26(1,23-1,29)$ & $1,08(1,05-1,10)$ & $1,17(1,14-1,19)$ \\
\hline \multicolumn{7}{|l|}{ Homens } \\
\hline \multicolumn{7}{|l|}{ Escolaridade } \\
\hline 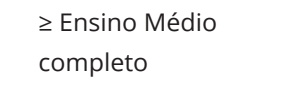 & 1,00 & 1,00 & 1,00 & 1,00 & 1,00 & 1,00 \\
\hline $\begin{array}{l}\text { Ensino Médio } \\
\text { incompleto }\end{array}$ & $0,72(0,67-0,77)$ & $0,93(0,87-1,00)$ & $0,69(0,65-0,74)$ & $0,91(0,86-0,96)$ & $0,78(0,74-0,82)$ & $0,98(0,94-1,03)$ \\
\hline $\begin{array}{l}\text { Ensino Fundamental } \\
\text { completo }\end{array}$ & $0,84(0,79-0,90)$ & $1,00(0,94-1,07)$ & $0,87(0,82-0,92)$ & $0,98(0,93-1,04)$ & $0,98(0,93-1,02)$ & $1,04(0,99-1,09)$ \\
\hline $\begin{array}{l}\text { Ensino Fundamental } \\
\text { incompleto }\end{array}$ & $1,29(1,22-1,38)$ & $1,06(0,99-1,12)$ & $1,39(1,32-1,47)$ & $1,06(1,01-1,12)$ & $1,56(1,49-1,63)$ & $1,09(1,04-1,13)$ \\
\hline Analfabeto & $1,61(1,51-1,71)$ & $1,07(1,00-1,14)$ & $1,67(1,58-1,76)$ & $1,06(1,00-1,12)$ & $1,78(1,70-1,87)$ & $1,08(1,03-1,12)$ \\
\hline \multicolumn{7}{|l|}{ Renda mensal (SM) ** } \\
\hline$>10,01$ & 1,00 & 1,00 & 1,00 & 1,00 & 1,00 & 1,00 \\
\hline$>3,0-10,0$ & $0,93(0,89-0,97)$ & $0,99(0,96-1,03)$ & $0,90(0,87-0,94)$ & $0,98(0,95-1,03)$ & $0,89(0,85-0,92)$ & $0,97(0,94-1,01)$ \\
\hline$<3,0$ & $1,00(0,96-1,04)$ & $1,02(0,98-1,07)$ & $0,90(0,87-0,94)$ & $1,02(0,98-1,05)$ & $0,85(0,82-0,88)$ & $0,96(0,93-0,99)$ \\
\hline \multicolumn{7}{|l|}{ Mulheres } \\
\hline \multicolumn{7}{|l|}{ Escolaridade } \\
\hline $\begin{array}{l}\geq \text { Ensino Médio } \\
\text { completo }\end{array}$ & 1,00 & 1,00 & 1,00 & 1,00 & 1,00 & 1,00 \\
\hline $\begin{array}{l}\text { Ensino Médio } \\
\text { incompleto }\end{array}$ & $1,22(1,14-1,32)$ & $1,37(1,28-1,48)$ & $1,06(0,99-1,11)$ & $1,20(1,14-1,27)$ & $1,12(1,07-1,18)$ & $1,24(1,19-1,29)$ \\
\hline $\begin{array}{l}\text { Ensino Fundamental } \\
\text { completo }\end{array}$ & $1,95(1,82-2,09)$ & $1,91(1,79-2,04)$ & $1,89(1,79-1,99)$ & $1,79(1,71-1,89)$ & $1,83(1,76-1,92)$ & $1,61(1,54-1,67)$ \\
\hline Analfabeto & $3,19(2,98-3,41)$ & $2,28(2,13-2,43)$ & $3,06(2,91-3,21)$ & $2,06(2,01-2,23)$ & $3,15(3,03-3,28)$ & $1,89(1,81-1,95)$ \\
\hline \multicolumn{7}{|l|}{ Renda mensal (SM) ** } \\
\hline$>10,01$ & 1,00 & 1,00 & 1,00 & 1,00 & 1,00 & 1,00 \\
\hline$>3,0-10,0$ & $1,26(1,21-1,30)$ & $1,30(1,26-1,34)$ & $1,20(1,16-1,24)$ & $1,25(1,21-1,29)$ & $1,14(1,10-1,17)$ & $1,18(1,15-1,22)$ \\
\hline$<3,0$ & $1,46(1,42-1,51)$ & $1,42(1,37-1,46)$ & $1,38(1,34-1,43)$ & $1,46(1,41-1,51)$ & $1,26(1,22-1,30)$ & $1,34(1,30-1,38)$ \\
\hline
\end{tabular}

IC95\%: intervalo de 95\% de confiança; SM: salários mínimos.

* Ajustado para sexo, idade, cor da pele e regiões do Brasil;

** Renda mensal em SM para o ano de 1998 ( $R \$ 130,00)$, 2003 ( $R \$ 240,00)$ e $2008(R \$ 415,00)$. 
primeira teoria, uma vez que tanto desfecho quanto exposição foram medidos de maneira semelhante nos inquéritos, e o mesmo padrão da associação manteve-se nos três anos estudados. Não há como avaliar a veracidade da segunda e quarta teorias. Portanto, os achados deste estudo vão ao encontro da teoria "materialista ou estruturalista".

Este trabalho tende a concordar com os argumentos propostos no modelo de Diderichsen et al. 45. Nesse modelo, a posição social que um indivíduo ocupa na sociedade seria o resultado de diversos mecanismos sociais, como a situação no mercado de trabalho e educação. $\mathrm{O}$ individuo em situação econômica mais desfavorável estaria mais exposto a uma série de fatores de risco, que poderiam fazer mal à sua saúde. Quando expostas a fatores de risco, as pessoas em pior posição socioeconômica teriam maior probabilidade de apresentar doença, devido a um diferencial de vulnerabilidade a que estão expostas. O indivíduo em pior situação econômica, estando doente, teria mais dificuldades de acesso a serviços de saúde, cuidados médicos e tratamentos necessários para a sua reabilitação.

Outras explicações para a associação entre hipertensão arterial e o nível socioeconômico têm sido sugeridas. Existiriam diferenças no acesso aos serviços de saúde, na distribuição de fatores de risco cardiovascular, na presença de outras comorbidades associadas à hipertensão arterial nos diferentes grupos, além de fatores ambientais 35 . Essas diferenças também seriam mediadas por tensões sociais, falta de apoio social e dificuldades de acesso a recursos públicos 31 .

Países de alta renda têm reduzido a prevalência de hipertensão arterial por meio de ações em políticas públicas, como a redução de sal em alimentos processados, com diagnóstico precoce e tratamentos disponíveis à população, além do monitoramento de outros fatores de risco para doenças cardiovasculares. De forma contrária, em países de baixa renda, como o Brasil, as prevalências de hipertensão arterial ainda apresentam crescimento. A hipertensão arterial, embora seja um fator de risco evitável, reflete em custos médicos elevados, como taxas de hospitalizações e gastos em medicamentos, principalmente em função de suas complicações, tais como: doença cerebrovascular, doença arterial coronariana, insuficiência cardíaca, insuficiência renal crônica e doença vascular de extremidades 1,46 .

Considerando que o tratamento da hipertensão arterial reduz significativamente a mortalidade e a morbidade por doença cardiovascular 1, é importante o conhecimento da prevalência deste fator de risco na população brasileira, bem como a identificação de grupos mais vulneráveis e prioritários para a elaboração de políticas públicas mais equânimes. Os dados utilizados neste estudo fazem parte de grandes inquéritos populacionais, sendo assim representativos da população brasileira. Tais estudos são fundamentais para o monitoramento das condições de saúde e do acesso a serviços de saúde em uma população.

Assim, o presente trabalho apontou o aumento das prevalências de hipertensão arterial nos períodos estudados, destacando a sua associação com baixos níveis de escolaridade, mulheres de baixa renda e nos homens com renda mais elevada.

\section{Colaboradores}

L. A. C. Lobo participou do planejamento, elaboração do estudo, revisão bibliográfica, análise de dados e redação do artigo. R. Canuto participou da elaboração do estudo e análise de dados. J. S. Dias-da-Costa participou da redação, análise de dados e revisão do artigo. M. P. Pattussi participou do planejamento, elaboração do estudo e revisão do artigo. 


\section{Referências}

1. Departamento de Atenção Básica, Secretaria de Atenção à Saúde, Ministério da Saúde. Diretrizes para o cuidado das pessoas com doenças crônicas nas redes de atenção à saúde e nas linhas de cuidado prioritárias. Brasília: Ministério da Saúde; 2013.

2. World Health Organization. A global brief on hypertension: silent killer, global public health crisis. World Health Day 2013. Geneva: World Health Organization; 2013.

3. Mendis S, Puska P, Norrving B. Global atlas on cardiovascular disease prevention and control: policies, strategies and interventions. Geneva: World Health Organization; World Heart Federation; World Stroke Organization; 2011.

4. Mittal BV, Singh AK. Hypertension in the developing world: challenges and opportunities. Am J Kidney Dis 2010; 55:590-8.

5. Picon RV, Fuchs FD, Moreira LB, Riegel G, Fuchs SC. Trends in prevalence of hypertension in Brazil: a systematic review with metaanalysis. Plos One 2012; 7:e48255.

6. Secretaria de Vigilância em Saúde, Ministério da Saúde. Vigitel Brasil 2014: Vigilância de Fatores de Risco e Proteção para Doenças Crônicas por Inquérito Telefônico. Ministério da Saúde, Secretaria de Vigilância em Saúde. Brasília: Ministério da Saúde; 2015.

7. Andrade SSA, Stopa SR, Brito AS, Chueri PS, Szwarcwald CL, Malta DC. Prevalência de hipertensão arterial autorreferida na população brasileira: análise da Pesquisa Nacional de Saúde, 2013. Epidemiol Serv Saúde 2015; 24:297-304.

8. Karlamangla AS, Singer BH, Williams DR, Schwartz JE, Matthews KA, Kiefe CI, et al. Impact of socioeconomic status on longitudinal accumulation of cardiovascular risk in young adults: the CARDIA Study (USA). Soc Sci Med 2005; 60:999-1015.

9. Schmidt MI, Duncan BB, Silva GA, Menezes AM, Monteiro CA, Barreto SM, et al. Doenças crônicas não transmissíveis no Brasil: carga e desafios atuais. Saúde no Brasil 4. Lancet 2011; 377:1949-61.

10. Veenstra G. Race, gender, class, sexuality (RGCS) and hypertension. Soc Sci Med 2013; 89:16-24.

11. Muniz LC, Cascaes AM, Wehrmeister FC, Martínez-Mesa J, Barros AJ, Menezes AM. Trends in self-reported arterial hypertension in Brazilian adults: an analysis of data from the Brazilian National Household Sample Survey, 1998-2008. Cad Saúde Pública 2012; 28:1599-607.

12. Instituto Brasileiro de Geografia e Estatística. Pesquisa Nacional por Amostra de Domicílios. Rio de Janeiro: Instituto Brasileiro de Geografia e Estatística; 2011.

13. Almeida MF, Barata RB, Monteiro CV, Silva ZP. Prevalência de doenças crônicas auto-referidas e utilização de serviços de saúde, PNAD/1998, Brasil. Ciênc Saúde Coletiva 2002; 7:743-56.
14. Travassos C, Oliveira EXG, Viacava F. Desigualdades geográficas e sociais no acesso aos serviços de saúde no Brasil: 1998 e 2003. Ciênc Saúde Coletiva 2006; 11:975-86.

15. Chrestani MAD, Santos IS, Matijasevich AM. Hipertensão arterial sistêmica auto-referida: validação diagnóstica em estudo de base populacional. Cad Saúde Pública 2009; 25:2395-406.

16. Lima-Costa MF, Peixoto SV, Firmo JOA. Validade da hipertensão arterial auto-referida e seus determinantes (projeto Bambuí). Rev Saúde Pública 2004; 38:637-64.

17. Selem SSC, Castro MA, César CLG, Marchioni DML, Fisberg RM. Validade da hipertensão autorreferida associa-se inversamente com escolaridade em brasileiros. Arq Bras Cardiol 2013; 100:52-9.

18. Mozaffarian D, Benjamin EJ, Go AS, Arnett DK, Blaha MJ, Cushman M, de Ferranti S, et al; American Heart Association Statistics Committee and Stroke Statistics Subcommittee. Heart disease and stroke statistics, 2015 update: a report from the American Heart Association. Circulation 2015; 131:e29-322.

19. Paim J, Travassos CMR, Almeida CM, Bahia L, Macinko J. O sistema de saúde brasileiro: história, avanços e desafios. Saúde no Brasil 1. Lancet 2011; 6736:60054-811-29.

20. Piccini RX, Facchini LA, Tomasi E, Siqueira FV, Silveira DS, Thumé E, et al. Promoção, prevenção e cuidado da hipertensão arterial no Brasil. Rev Saúde Pública 2012; 46:543-50.

21. Passos VMA, Assis TD, Barreto SM. Hipertensão arterial no Brasil: estimativa de prevalência a partir de estudos de base populacional. Epidemiol Serv Saúde 2006; 15:35-45.

22. Pinheiro RS, Viacava F, Travassos C, Brito AS. Gênero, morbidade, acesso e utilização de serviços de saúde no Brasil. Ciênc Saúde Coletiva 2002; 7:687-707.

23. Arber S, Cooper H. Gender differences in health in later life: the new paradox? Soc Sci Med 1999; 48:61-76.

24. Denton M, Prus S, Walters V. Gender differences in health: a Canadian study of the psychosocial, structural and behavioural determinants of health. Soc Sci Med 2004; 58:2585-600.

25. Macintyre S, Hunt K. Socioeconomic position, gender and health: how do they interact? J Health Psychol 1997; 2:315-34.

26. Moreira JPL, Moreira JP, Moraes JR, Luiz RR. Prevalence of self-reported systemic arterial hypertension in urban and rural environments in Brazil: a population-based study. Cad Saúde Pública 2013; 29:62-72.

27. Ross CE, Bird CE. Sex stratification and health lifestyle: Consequences for men's and women's perceived health. J Health Soc Behav 1994; 35:161-78. 
28. Brummett BH, Babyak MA, Siegler IC, Shanahan M, Harris KM, Elder GH, et al. Nationally representative US young adult sample systolic blood pressure, socioeconomic status, and biobehavioral risk factors in a nationally representative US young adult sample. Hypertension 2011; 58:161-6.

29. Dalstra JA, Kunst AE, Geurts JJ, Frenken FJ, Mackenbach JP. Trends in socioeconomic health inequalities in the Netherlands, 19811999. J Epidemiol Community Health 2002; 56:927-34

30. De Gaudemaris R, Lang T, Chatellier G, Larabi L, Lauwers-Cancès V, Maître A, et al. Socioeconomic inequalities in hypertension prevalence and care: the IHPAF Study. Hypertension 2002; 39:1119-25.

31. Luepker RV, Rosamond WD, Murphy R, Sprafka JM, Folsom AR, McGovern PG, et al. Socioeconomic status and coronary heart disease risk factor trends. The Minnesota Heart Survey. Circulation 1993; 88:2172-9.

32. Luoto R, Pekkanen J, Uutela A, Tuomilehto J. Cardiovascular risks and socioeconomic status: differences between men and women in Finland. J Epidemiol Community Health 1994; 48:348-54.

33. Townsend P, Davidson N, Whitehead M. Inequalities in health: the Black Report/the Health Divide. London: Penguin UK; 1992.

34. Tormo MJ, Navarro C, Chirlaque MD, Barber $\mathrm{X}$. Validation of self diagnosis of high blood pressure in a sample of the Spanish EPIC cohort: overall agreement and predictive values. EPIC Group of Spain. J Epidemiol Community Health 2000; 54:221-6.

35. Tyroler HA. Socioeconomic status in the epidemiology and treatment of hypertension. Hypertension 1989; 13:93-7.

36. Van Rossum CT, van de Mheen H, Witteman JC, Hofman A, Mackenbach JP, Grobbee DE. Prevalence, treatment, and control of hypertension by sociodemographic factors among the Dutch elderly. Hypertension 2000; 35:814-21.

37. Barros MBA, Bergamo F, Zanchetta LM, César CLG. Tendências das desigualdades sociais e demográficas na prevalência de doenças crônicas no Brasil, PNAD: 2003-2008. Ciênc Saúde Coletiva 2011; 16:3755-68.
38. Dias da Costa JS, Barcellos FC, Sclowitz ML, Sclowitz IKT, Castanheira M, Olinto MTA, et al. Prevalência de hipertensão arterial em adultos e fatores associados: um estudo de base populacional urbana em Pelotas, Rio Grande do Sul, Brasil. Arq Bras Cardiol 2007; 88:59-65.

39. Ferreira SR, Moura EC, Malta DC, Sarno F. Frequency of arterial hypertension and associated factors: Brazil, 2006. Rev Saúde Pública 2009; 43 Suppl 2:98-106.

40. Fuchs FD, Moreira LB, Moraes RS, Bredemeier M, Cardozo SC. Prevalência de hipertensão arterial sistêmica e fatores associados na região urbana de Porto Alegre. Estudo de base populacional. Arq Bras Cardiol 1994; 63:473-9.

41. Hartmann M, Dias-da-Costa JS, Olinto MTA, Pattussi MP, Tramontini A. Prevalência de hipertensão arterial sistêmica e fatores associados: um estudo de base populacional em mulheres no Sul do Brasil. Cad Saúde Pública 2007; 23:1857-66.

42. Jardim PCBV, Moreira HG, Monego ET, Gondim MRP, Vitorino PVO, Souza WKSB, et al. Hipertensão Arterial e alguns fatores de risco em uma capital brasileira. Arq Bras Cardiol 2007; 88:452-7.

43. Marins VM, Almeida RM, Pereira RA, Sichieri R. The association between socioeconomic indicators andcardiovascular disease risk factors in Rio de Janeiro, Brasil. J Biosoc Sci 2007; 39:221-9.

44. Kaplan G, Keil JE. Social factors and cardiovascular disease: review of the literature. Circulation 1993; 88:1973-88.

45. Diderichsen F, Evans T, Whitehead M. The social basis of disparities in health. In: Evans T, Whitehead M, Diderichsen F, Bhuiya A, Wirth $\mathrm{M}$, editors. Challenging inequities in health: from ethics to action. New York: Oxford University Press; 2001. p. 12-23.

46. V Diretrizes Brasileiras de Hipertensão Arterial. Arq Bras Cardiol 2007; 89:e24-79. 


\section{Abstract}

The objectives were to analyze the prevalence of arterial hypertension reported by Brazilian adults over 20 years of age and verify associated socioeconomic variables in three time periods. The data are from the Brazilian National Household Sample Survey (PNAD) conducted by the Brazilian Institute of Geography and Statistics (IBGE) in 1998 (196,439 participants), 2003 $(231,921)$, and $2008(250,664)$. The outcome was self-reported arterial hypertension. The principal exposures were income in Reais and schooling in years. Data analysis used Poisson regression with robust variance with control for complex samples. Higher prevalence of arterial hypertension was associated with lower schooling, regardless of the survey year and gender. Low income was associated with higher prevalence of arterial hypertension, regardless of years of schooling, in the overall sample and in women. In men, this effect was not observed in 1998 and 2003. In 2008, high-income men showed higher prevalence of arterial hypertension, suggesting effect modification. Thus, the current study pointed to an increase in prevalence rates for arterial hypertension in the three periods, highlighting the inverse association with socioeconomic factors.

Hypertension; Health Inequalities; Health Surveys

\section{Resumen}

El estudio analiza las prevalencias de hipertensión arterial, referidas por adultos brasileños con más de 20 años, y verifica las variables socioeconómicas asociadas durante tres periodos de tiempo. Los datos provienen de la Encuesta Nacional por Muestra de Domicilios (PNAD), realizada por el Instituto Brasileño de Geografía y Estadística (IBGE), durante los años 1998 (196.439 participantes), 2003 (231.921) y 2008 (250.664). El resultado fue la hipertensión arterial mencionada. Las exposiciones principales eran renta en reales y escolaridad en años de estudios. El análisis de los datos fue realizado usándose la regresión de Poisson con variancia robusta con control para muestras complejas. Mayores prevalencias de hipertensión arterial se encontraron en personas con menor escolaridad, independientemente de los años estudiados y del sexo. La baja renta estuvo asociada a mayores prevalencias de hipertensión arterial, independientemente de los años estudiados en la muestra total y en las mujeres. Entre los hombres, ese efecto no se verificó durante los años 1998 y 2003. Ya en 2008, hombres con renta alta presentaron mayores prevalencias de hipertensión arterial, sugiriendo la modificación de efecto. De esta forma, el presente trabajo apuntó el aumento de las prevalencias de hipertensión arterial durante los períodos estudiados, destacando su asociación negativa con aspectos socioeconómicos.

Hipertensión; Desigualdades en la Salud;

Encuestas Epidemiológicas
Recebido em 03/Mar/2016

Versão final reapresentada em 05/Jul/2016

Aprovado em 28/Jul/2016 